Original Research Paper

\title{
Hepatoprotective Impact of TLR9 Antagonist ODN 2088 Against Carbon Tetrachloride $\left(\mathrm{CCl}_{4}\right)$-Induced Hepatic Injury
}

\author{
Badr Alzahrani \\ Department of Clinical Laboratory Sciences, College of Applied Medical Sciences, Jouf University, Saudi Arabia
}

Article history

Received: 11-11-2019

Revised: $30-12-2019$

Accepted: 04-01-2020

Email: baalzahrani@ju.edu.sa

\begin{abstract}
The main objective of this study is to evaluate the hepatoprotective impact of TLR9 antagonist ODN 2088 against carbon tetrachloride $\left(\mathrm{CCl}_{4}\right)$-induced acute liver injury. In this study, 24 mice were grouped into four groups. $\mathrm{CCl}_{4}$ was introduced in all groups except for the control group intraperitoneally after pretreatment with ODN 2088 and silymarin for seven days. To assess the liver injury, serum alanine Aminotransferase (ALT) and aspartate Aminotransferase (AST) were estimated and histopathological changes in the liver tissue were investigated. Expression of pro-inflammatory cytokines including TNF- $\alpha$ and IL-6 were measured. It was observed that the serum levels of biochemical parameters such as ALT and AST were remarkably elevated in mice treated only with $\mathrm{CCl}_{4}$ compared with the control mice (treated with corn oil). This increase in levels of ALT and AST was remarkably diminished by pretreatment with ODN 2088. The hepatoprotective impact of ODN 2088 on $\mathrm{CCl}_{4}$-intoxicated mice was verified by histopathological studies. ODN2088 pretreatment inhibited liver inflammation by diminishing the liver expression of $\mathrm{CCl}_{4}$-induced activity of TNF- $\alpha$ and IL6 . In conclusion, TLR9 antagonist, ODN 2088, protected the mice against $\mathrm{CCl}_{4}$-induced liver damage and inflammation by down-regulating the expression of pro-inflammatory cytokines.
\end{abstract}

Keywords: Liver Injury, TLR9, ODN 2088, $\mathrm{CCl}_{4}$

\section{Introduction}

Acute liver injury can develop when the liver is exposed to various hepatotoxic factors such as toxic chemicals, pathogen infections and drugs. It is strongly associated with development of oxidative stress and inflammation in the liver tissue (Lee et al., 2019). Continuous exposure to hepatotoxic factors will induce the development of liver fibrosis, cirrhosis and Hepatocellular Carcinoma (HCC) (Lee et al., 2019). Carbon tetrachloride $\left(\mathrm{CCl}_{4}\right)$ is a toxin that is widely used in animal models to induce hepatotoxicity. The use of $\mathrm{CCl}_{4}$ to induced liver injury has helped in understanding the mechanism of liver injury development as well as evaluating the potentials drugs. When $\mathrm{CCl}_{4}$ is introduced in the body, cytochrome P450 enzymes metabolise it, resulting in production of trichloromethyl radicals. Production of these radicals involves the Kupffer cell activation and releasing of pro-inflammatory cytokines, such as Tumour Necrosis Factor- $\alpha$ (TNF- $\alpha$ ) and interleukin6 (IL-6) (Chiu et al., 2018). In addition, necrosis and apoptosis are two processes that regenerate in the liver tissue prior to $\mathrm{CCl}_{4}$ introduction, which can result in stimulation of hepatic inflammation (Yang et al., 2015) and Extracellular Matrix (ECM) accumulation (Chiu et al., 2018). Further, $\mathrm{CCl}_{4}$ can stimulate the oxidative degradation of cellular lipids and lipid peroxidation (Chiu et al., 2018).

Toll-Like Receptors (TLRs) are known as type I transmembrane proteins that are produced by parenchymal and non-parenchymal cells of the liver. Many studies have shown that TLRs are involved in acute and chronic liver diseases. The hepatocellular necrosis that occurs during acute liver injury can result in releasing cellular constituents known as DamageAssociated Molecular Patterns (DAMPs). Examples of DAMPs include High Mobility Group Box-1 protein (HMGB1), DNA, RNA and S100 proteins. Binding of DAMP components with TLRs stimulates the production of pro-inflammatory cytokines such as TNF- $\alpha$ and IL-6 via activation of Nuclear factor kappa-light-enhancer of activated B cells (NF- $\kappa \mathrm{B}$ ) (Wang et al., 2019).

It has been shown that the DNA released from apoptotic hepatocytes during liver injury is triggered by 
TLR9 (Bakker et al., 2015). DNA released in the circulation during severe liver injury can stimulate systemic inflammation by binding with TLR9 (Bakker et al., 2015). One study revealed that TLR9 can also recognise microbial DNA rich in $\mathrm{CpG}$ motifs. This recognition can increase the production of TNF- $\alpha$, IL- 6 and chemokines by liver tissue. CpG DNA can enhance the production of TNF- $\alpha$ through the TLR9/My88 pathway, which results in a severe acute liver injury in D-galactosamine-sensitised mice (Yi et al., 2006). Another study determined that hepatotoxicity was dependent on the TLR9 pathway in mice treated with acetaminophen to induce liver injury (Imaeda et al., 2009). This toxin induces necrosis and apoptosis of hepatocytes, which can result in activation of innate immunity. The released DNA from apoptotic hepatocytes is involved in TLR9 activation and in turn increases the expression of IL-1 beta and IL-18 (Imaeda et al., 2009). The current study was performed to validate the hepatoprotective effects of ODN 2088 (TLR9 antagonist) in acute liver damage model caused by $\mathrm{CCl}_{4}$. ODN 2088 is a short nucleotide sequence that inhibits the activity of TLR9 by preventing the binding of TLR9 with its ligands.

\section{Materials and Methods}

\section{Materials}

$\mathrm{CCl}_{4}$ and corn oil were purchased from Sigma-Aldrich (MO, US) and TLR9 antagonist CpG ODN2088: 5'tcctggcggggaagt-3' was provided by InvivoGen (CA, US).

\section{Animals and Treatments}

One week before the experiments, the mice were maintained in standard cages in a temperature- and humidity-controlled room with a 12-h light-dark cycle. Four-to-six-week old male BALB/c mice were divided into four groups ( $\mathrm{n}=6$ mice per group). Group I mice were given normal food and water for seven days and received a single intraperitoneal (ip) injection of corn oil on the seventh day. Group II mice were given normal food and water and received an ip injection of $\mathrm{CCl}_{4}$ mixed with corn oil $\left(1: 19(\mathrm{v} / \mathrm{v}), 0.5 \mathrm{~mL} / \mathrm{kg}\right.$ of $\left.\mathrm{CCl}_{4}\right)$. Group III were administrated TLR9 antagonist ODN2088 via ip injection for seven days $(50 \mathrm{ug} / 20 \mathrm{~g}$ body weight resuspended in endotoxin-free water), then $\mathrm{CCl}_{4}$ was ip injected on the seventh day. Group IV mice were orally treated with silymarin $(0.2 \mathrm{~g} / \mathrm{kg})$ for seven days and the $\mathrm{CCl}_{4}$ mixture was introduced on the seventh day. On the eighth day, all mice were euthanised. Mice liver tissue was harvested and parts of the livers taken for histological studies. Blood was collected directly from the mice hearts and centrifuged for $10 \mathrm{~min}$ at $1,000 \times \mathrm{g}$ to isolate sera and then kept at $4^{\circ} \mathrm{C}$ for biochemical measurements. The Local Committee of Bioethics (LCBE), Jouf University (Sakaka, Saudi Arabia) approved the protocols of the animal experiments.

\section{Histological Examination of Liver Tissue}

The liver tissues were fixed in $10 \%$ formalin, then deparaffinised in xylene and dehydrated in ethanol at $70 \%$ and $100 \%$. Liver tissues were then embedded and cut $5 \mathrm{um}$ thick. For photomicroscopic examination, Hematoxylin and Eosin (H\&E) was performed. Images of sections were taken by the Olympus DP2-BSW application software (Olympus Imaging System, Tokyo, Japan) and ImageJ was applied for image quantification.

\section{Measurements of Serum ALT and AST}

After mice blood centrifugation, measurements of the serum level of aspartate Aminotransferase (AST) and alanine Aminotransferase (ALT) were taken via a clinical chemistry analyser (Beckman Counter LX20, US).

\section{Immunohistochemistry}

IHC was performed to study the modification in protein expressions in the liver tissue. Liver tissues were cut into 5 um sections and deparaffinised, rehydrated and treated with $3 \%$ hydrogen peroxide. PBS washed the liver sections three times for five minutes each. To block nonspecific proteins in the liver tissue, sections were incubated in $10 \%$ BSA for 10 min. Primary antibodies diluted in a washing buffer were incubated with liver sections for $24 \mathrm{~h}$. The primary antibodies used were TNF$\alpha$ (1:100 dilution, Sant Cruz Biotechnology, Sant Cruz, US) and IL-6 antibody (diluted at 1:100 in PBS, Abcam, Cambridge, UK). Following that, sections were washed by PBS three time for five minutes each and secondary antibodies were incubated with the sections for $1 \mathrm{~h}$ at room temperature. Then, sections were washed three times and incubated with a diaminobenzidine tetrahydrochloride (DAKO, Glostrup, Denmark) solution for $10 \mathrm{~min}$ and counterstained. Images of the sections were taken with the Olympus DP2-BSW application software (Olympus Imaging System, Tokyo, Japan) and ImageJ was applied for image quantification.

\section{Statistical Analysis}

The data are presented as mean \pm Standard Error of the Mean (SEM). GraphPad Prism software was used to analyse the quantitative data. As this study was performed in groups, one-way analysis of variance and Tukey's multiple group comparison tests were applied to analyse the data. Results were regarded significant if $p$ values were less than 0.05 .

\section{Results}

Effects of ODN 2088 on the Serum Level of ALT and $A S T$

Secretion of ALT and AST from liver tissue increases during liver injury. These two enzymes are used as 
chemical biomarkers for acute liver injury or hepatotoxicity. In this study, measurements of ALT and AST were carried out to evaluate the liver damage in the mice groups. In comparison to controls, $\mathrm{CCl}_{4}$-administrated mice revealed a significant increase in the serum level of ALT and AST $(p<0.01)$. However, pre-administration of ODN 2088 (ip) was able to prevent the elevated ALT and AST in serum, as shown in Fig. 1. Also, the mice group pretreated with silymarin, used as a positive control, prohibited the increased serum level of ALT and AST (Fig. 1).

\section{Histopathological Examination}

H\&E staining was performed to evaluate the changes in the liver architecture and morphology in mice groups. The sections for the control group showed a normal liver architecture (Fig. 2A). However, severe damage in the liver tissue of $\mathrm{CCl}_{4}$-administrated mice group was revealed in comparison with the control group associated with increased inflammation (Fig. 2B). Further, preadministration of ODN 2088 prior ip infection of $\mathrm{CCl}_{4}$ prevented liver damage and inflammation (Fig. 3C and 3D).

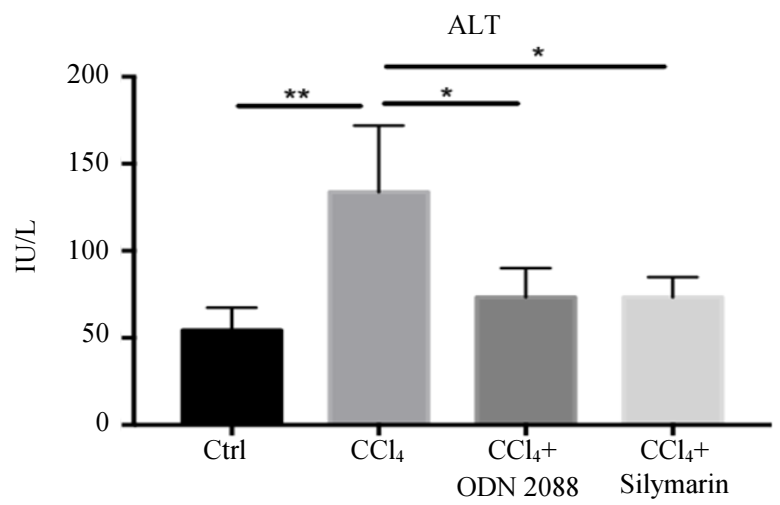

ODN 2088 Diminished Protein Expression of TNF$\alpha$ and IL-6

To understand the role of ODN 2088 in the expression of pro-inflammatory cytokines on damaged liver tissue, an immunohistochemical analysis was used and quantitative assessments of the protein expression of TNF- $\alpha$ and IL-6 were evaluated (Fig. 3 and 4). The quantitative assessments revealed that $\mathrm{CCl}_{4}$-treated mice developed a significant elevation in the protein expression of TNF- $\alpha$ and IL-6 compared with the control group ( $p<0.01$ and $p<0.05$, respectively). However, a remarkable fall in the TNF- $\alpha$ and IL- 6 positive area were detected in mice that were pretreated with ODN 2088 in comparison with $\mathrm{CCl}_{4}$-injected mice $(p<0.01$ for both comparisons). Given that TLR-9 plays an important role in the regulation of TNF- $\alpha$ and IL- 6 , it appears that the protective role of ODN 2088 during acute liver injury occurs via inhibiting the activity of TLR-9 and its role in stimulating the expressions of TNF- $\alpha$ and IL- 6 .

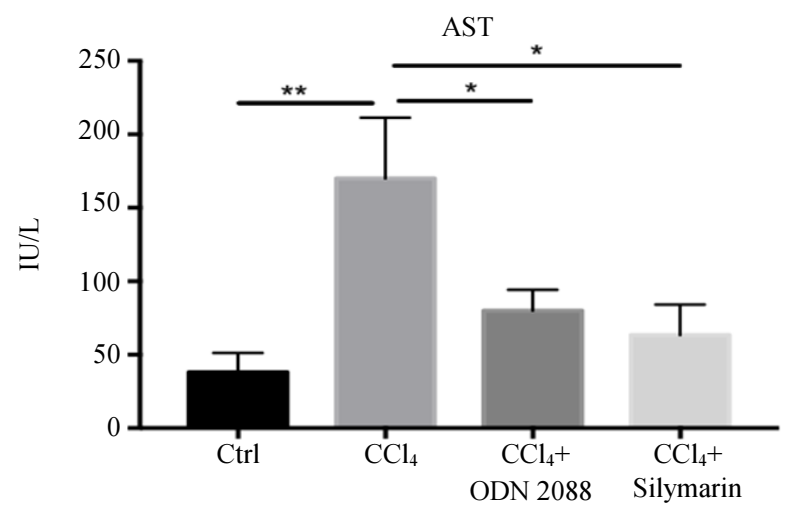

Fig. 1: Effects of TLR9 antagonist ODN 2088 on levels of serum enzymes ALT and AST in CCl4-induced liver damage in mice (n= 6). (A) Serum ALT level and; (B) serum AST level. Values are presented as mean \pm Standard Error of the Mean (SEM). Statistical significance was evaluated by one-way ANOVA $(* p \leq 0.05, * * p \leq 0.01)$
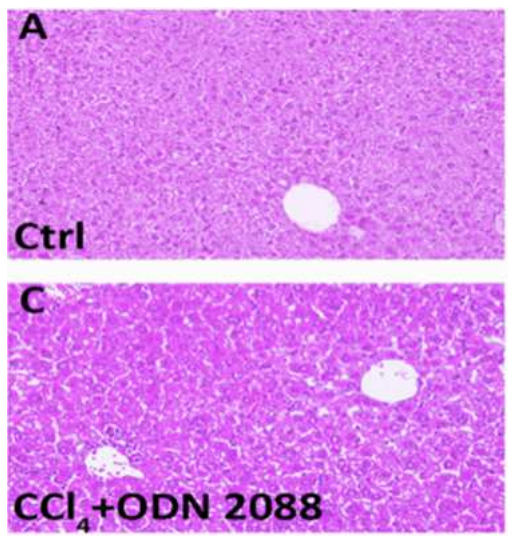
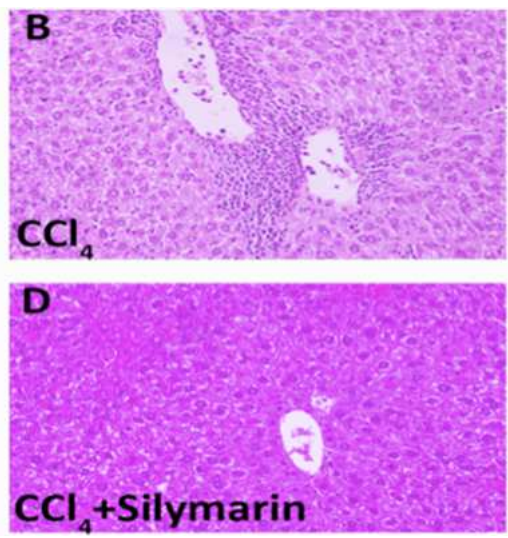

Fig. 2: $\mathrm{H} \& \mathrm{E}$ analysis of liver tissue after $\mathrm{CCl}_{4}$ intoxication in mice, original magnification $\times 20$. (A) Control mice group; (B) $\mathrm{CCl}_{4}$ treated mice; (C) mice pretreated with $\mathrm{ODN} 2088$ and then treated with $\mathrm{CCl}_{4}$; (D) positive control mice pretreated with silymarin followed by $\mathrm{CCl}_{4}$ treatment 

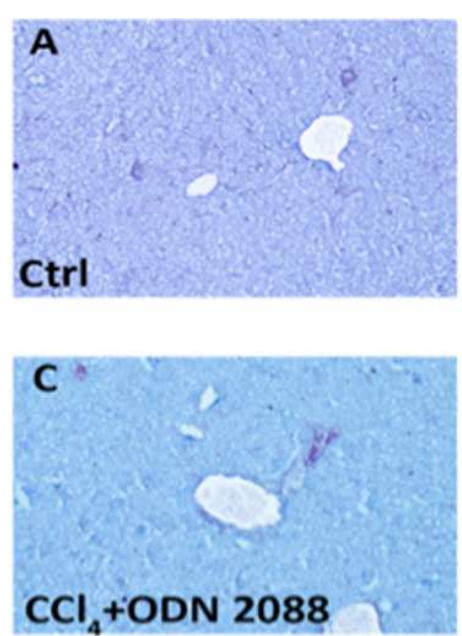
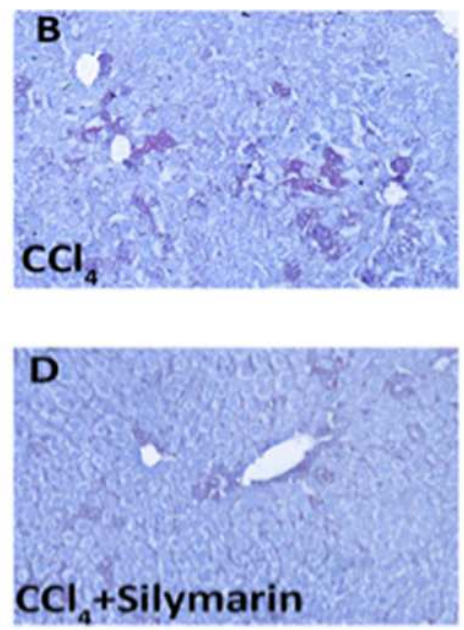

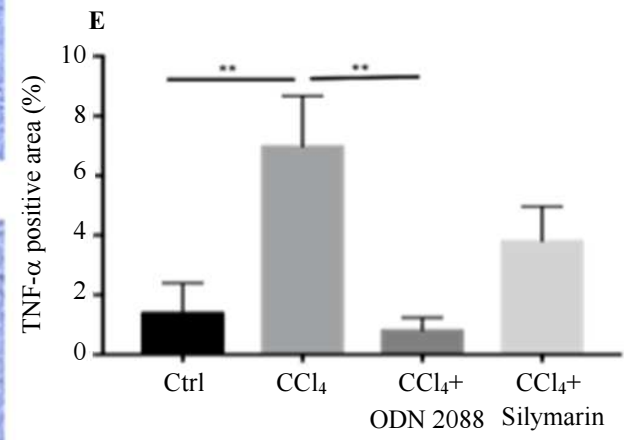

Fig. 3: Immunohistological analysis of hepatic TNF- $\alpha$ protein expression. (A) Control mice group; (B) $\mathrm{CCl}_{4}$-treated mice; (C) mice pretreated with ODN 2088 and then treated with $\mathrm{CCl}_{4}$; (D) positive control mice pretreated with silymarin followed by $\mathrm{CCl}_{4}$ treatment; (E) percentage of TNF- $\alpha$ positive area in mice liver. Values are presented as mean \pm Standard Error of the Mean (SEM). Statistical significance was determined by one-way ANOVA $(* * p \leq 0.01)$
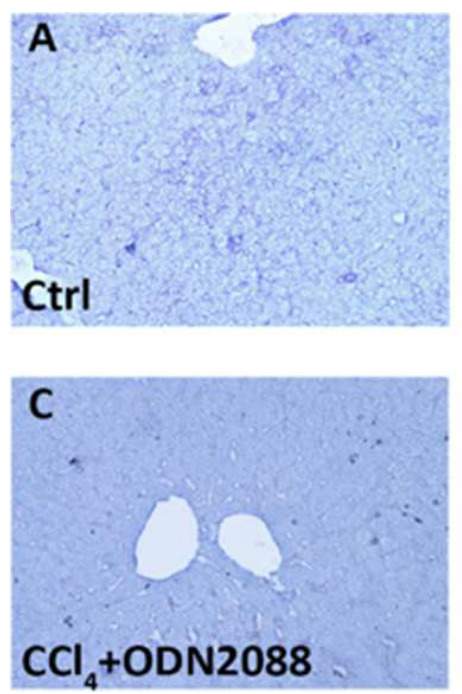
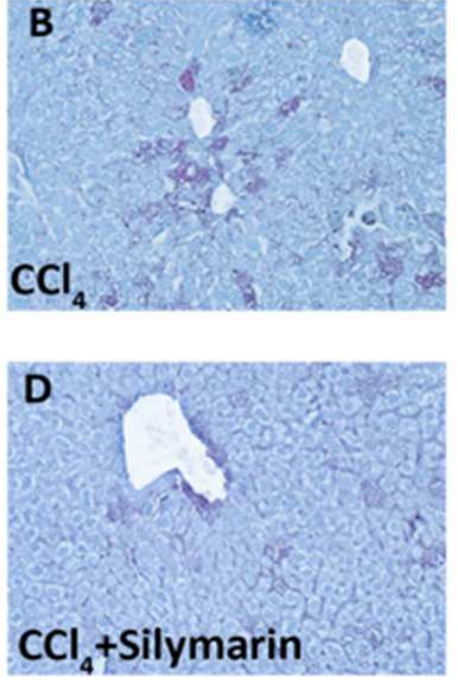

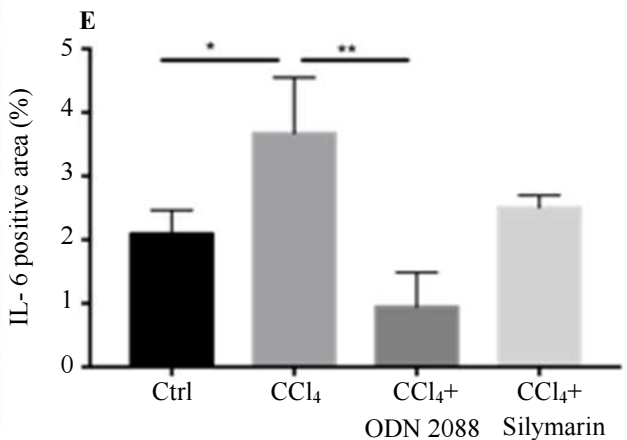

ODN 2088 Silymarin

Fig. 4: Immunohistological analysis of hepatic IL-6 protein expression. (A) Control mice group; (B) $\mathrm{CCl}_{4}$-treated mice; (C) mice pretreated with ODN 2088 and then treated with $\mathrm{CCl}_{4}$; (D) positive control mice pretreated with silymarin followed by $\mathrm{CCl}_{4}$ treatment; (E) percentage of IL-6 positive area in mice liver. Data are presented as mean \pm Standard Error of the Mean (SEM). Statistical significance was determined by one-way ANOVA $\left(* p \leq 0.05,{ }^{* *} p \leq 0.01\right)$

\section{Discussion}

This study demonstrates the hepatoprotective effects of TLR9 antagonist ODN 2088 against $\mathrm{CCl}_{4}$-induced acute liver damage in mice. $\mathrm{A} \mathrm{CCl}_{4}$ solvent is commonly used as a potent inducer of acute liver injury in animal experiments (Lee et al., 2019; Bak et al., 2016). $\mathrm{CCl}_{4}$ intoxication is beneficial for studying the hepatoprotective actions of potent therapeutic agents (Huang et al., 2012). Because $\mathrm{CCl}_{4}$ solvent is lipophilic, it can be easily uptaken by liver tissue and can cross the cell membranes and generate trichloromethyl peroxyl radicals that play a key role in liver injury progression (Yang et al., 2015).
ALT and AST are two enzymes involved in liver function. During liver injury, the serum level of these enzymes is elevated. Increases in the level of these enzymes are used as indicators of injured liver tissue (Chiu et al., 2018). They are released from damaged hepatocytes into the blood circulation and levels can be useful in indicating the severity of the liver injury. In this study, ALT and AST activities were significantly elevated in mice treated with $\mathrm{CCl}_{4}$. In contrast, the increase in the levels of ALT and AST was remarkably diminished by pretreatment with ODN 2088 as well as silymarin (Fig. 1A and 1B). According to these findings, it 
appears that the TLR9 antagonist ODN 2088 inhibited the hepatotoxicity induced by $\mathrm{CCl}_{4}$. The hepatoprotective effect of ODN 2088 was confirmed via an H\&E analysis.

In this current study, an H\&E analysis demonstrated that liver injury was accompanied by hepatic inflammation after $\mathrm{CCl}_{4}$ treatment, which did not appear in the livers of the control mice. However, the injury score and hepatocellular inflammation was decreased by treatment with ODN 2088 (Fig. 2). These results confirm the data obtained after serum ALT and AST measurements.

Hepatocellular necrosis and apoptosis are two processes that regenerate in liver tissue prior to the $\mathrm{CCl}_{4}$ introduction that can result in stimulation of hepatic inflammation (Yang et al., 2015). In the $\mathrm{CCl}_{4}$ model of mice, hepatic inflammation is believed to be the major pathological cause of acute liver injury (Hu et al., 2014). Necrotic and apoptotic hepatocytes release molecules known as DAMPs, which are significantly involved in the stimulation of liver inflammation and production of inflammatory factors (Matzinger, 2002). Examples of DAMPs released from hepatocytes include DNAs, RNAs, nuclear and cytosolic proteins and mitochondrial compounds (Matzinger, 2002; Chen et al., 2011; Rock et al., 2011; Kono and Rock, 2008).

The released DAMPs act as immunostimulatory molecules released from damaged hepatocytes triggered by TLRs, leading to elevated expression of proinflammatory factors (Bakker et al., 2015). Further, TLR9 can also recognise the CpG-rich DNA sourced from microbes during liver disorders. This recognition can lead to increased production of TNF- $\alpha$, IL- 6 and chemokines by hepatic tissue through activation of the TLR9/My88 pathway (Yi et al., 2006). TNF- $\alpha$ and IL-6 cytokines play a fundamental role during hepatic damage and inflammation in many liver diseases ( $\mathrm{Na}$ et al., 2015). In addition, expression of IL-1 beta and IL-18 were induced because of interactions of DNA from apoptotic hepatocytes and TLR9 in the mice model of hepatotoxicity induced by acetaminophen (Imaeda et al., 2009).

In this current study, ODN 2088 pretreatment significantly diminished the liver expression of $\mathrm{CCl}_{4}$ induced activity of TNF- $\alpha$ and IL-6 (Fig. 3 and 4). This is consistent with a previous report by (Shaker et al., 2016) which revealed that the use of TLR9 antagonist COV08-0064 decreased the expression of cytokines such as TNF- $\alpha$ and IL-6 in the Ischemia-Reperfusion (I/R) mice model (Shaker et al., 2016). COV08-0064 decreased the phosphorylation and activation of TLR9mediated pathways such as p38 MAPK, ERK and JNK (Shaker et al., 2016). Thus, it is suggested that the protective role of ODN 2088 during liver damage is regulated by inhibiting the expression of pro-inflammatory cytokines and therefore hepatic inflammation. In another study, it was revealed that blockage of TLR9 has a significant effect on reducing liver injury and inflammation by inhibiting the activation of TLR9/NF- $\kappa$ B pathway in neutrophils (Marques et al., 2015).

\section{Conclusion}

In conclusion, this study showed that the TLR9 antagonist ODN 2088 played a fundamental role in preventing the development of $\mathrm{CCl}_{4}$-induced liver injury in mice. It was shown that the blockage of TLR9 was associated with reduced serum ALT and AST. The protective impacts of ODN 2088 were attributable to down-regulation of the expression levels of hepatic TNF- $\alpha$ and IL-6, cytokines that stimulate liver inflammation.

\section{Acknowledgement}

The author is deeply grateful to Professor Ahmed Tantawy from Tanat University, Egypt for his assistance in the designing of the in vivo experiments.

\section{Ethics}

This article is original and contains unpublished material. The corresponding author confirms that all of the other authors have read and approved the manuscript and no ethical issues involved

\section{References}

Bak, J., N.K. Je, H.Y. Chung, T. Yokozawa and S. Yoon et al., 2016. Oligonol Ameliorates $\mathrm{CCl}_{4^{-}}$ induced liver injury in rats via the NF-Kappa $\mathrm{B}$ and MAPK signaling pathways. Oxidative Med. Cell. Longevity. DOI: 10.1155/2016/3935841

Bakker, P.J., A.M. Scantlebery, L.M. Butter, N. Claessen and G.J. Teske et al., 2015. TLR9 mediates remote liver injury following severe renal ischemia reperfusion. PloS One, 10: e0137511-e0137511. DOI: 10.1371 /journal.pone.0137511

Chen, C.F., W.C. Lee, H.I. Yang, H.C. Chang and C.L. Jen et al., 2011. Changes in serum levels of HBV DNA and alanine aminotransferase determine risk for hepatocellular carcinoma. Gastroenterology, 141: 1240-1248. DOI: 10.1053/j.gastro.2011.06.036

Chiu, Y., S. Chou, C. Chiu, C. Kao and K. Wu et al., 2018. Hepatoprotective effect of the ethanol extract of polygonum orientale on carbon tetrachloride-induced acute liver injury in mice. J. Food Drug Anal., 26: 369-379. DOI: 10.1016/j.jfda.2017.04.007

Hu, L., L. Li, D. Xu, X. Xia and R. Pi et al., 2014. Protective effects of neohesperidin dihydrochalcone against carbon tetrachloride-induced oxidative damage in vivo and in vitro. Chem. Biol. Int., 213: 51-59. DOI: 10.1016/j.cbi.2014.02.003

Huang, H.L., Y.J. Wang, Q.Y. Zhang, B. Liu and F.Y. Wang et al., 2012. Hepatoprotective effects of baicalein against $\mathrm{CCl}_{4}$-induced acute liver injury in mice. World J. Gastroenterol, 18: 6605-6613. DOI: 10.3748/wjg.v18.i45.6605 
Imaeda, A.B., A. Watanabe, M.A. Sohail, S. Mahmood and M. Mohamadnejad et al., 2009. Acetaminopheninduced hepatotoxicity in mice is dependent on TLR9 and the $\mathrm{Nalp}^{3}$ inflammasome. J. Clin. Invest., 119: 305-314. DOI: $10.1172 / \mathrm{JCI} 35958$

Kono, H. and K.L. Rock, 2008. How dying cells alert the immune system to danger. Nature Rev. Immunol., 8: 279-289. DOI: $10.1038 /$ nri2215

Lee, Y., I. Cho, J. Kim, M. Lee and S. Ku et al., 2019. Hepatoprotective effects of blue honeysuckle on $\mathrm{CCl}_{4}$-induced acute liver damaged mice. Food Sci. Nutrit., 7: 322-338. DOI:10.1002/fsn3.893

Marques, P.E., A.G. Oliveira, R.V. Pereira, B.A. David and L.F. Gomides et al., 2015. Hepatic DNA deposition drives drug-induced liver injury and inflammation in mice. Hepatology, 61: 348-360. DOI: $10.1002 /$ hep. 27216

Matzinger, P., 2002. The danger model: A renewed sense of self. Science, 296: 301-305. DOI: $10.1126 /$ science. 1071059

Na, T.Y., Y.H. Han, N.L. Ka, H.S. Park and Y.P. Kang et al., 2015. 22-S-Hydroxycholesterol protects against ethanol-induced liver injury by blocking the auto/paracrine activation of MCP-1 mediated by LXRalpha. J. Pathol., 235: 710-720. DOI: 10.1002 /path.4494
Rock, K.L., J.J. Lai and X. Kono, 2011. Innate and adaptive immune responses to cell death. Immunol. Rev., 243: 191-205. DOI: 10.1111/j.1600-065X.2011.01040.x

Shaker, M.E., B.N. Trawick and W.Z. Mehal, 2016. The novel TLR9 antagonist COV08-0064 protects from ischemia/reperfusion injury in non-steatotic and steatotic mice livers. Biochem. Pharmacol., 112: 90-101. DOI: 10.1016/j.bcp.2016.05.003

Wang, M., J. Niu, L. Ou, B. Deng and Y. Wang et al., 2019. Zerumbone protects against carbon tetrachloride $\left(\mathrm{CCl}_{4}\right)$-induced acute liver injury in mice via inhibiting oxidative stress and the inflammatory response: Involving the TLR4/NFkappaB/COX-2 Pathway. Molecules, 22: E1964-E1964. DOI: 10.3390/molecules24101964

Yang, B., X. Zhang, S. Guan and Z. Hua, 2015. Protective Effect of procyanidin $\mathrm{B}_{2}$ against $\mathrm{CCl}_{4^{-}}$ Induced acute liver injury in mice. Molecules, 20: 12250-12265. DOI: 10.3390/molecules200712250

Yi, A.K., H. Yoon, J.E. Park, B.S. Kim and H.J. Kim et al., 2006. CpG DNA-mediated induction of acute liver injury in D-galactosamine-sensitized mice: The mitochondrial apoptotic pathway-dependent death of hepatocytes. J. Biol. Chem., 281: 15001-15012. DOI: $10.1074 /$ jbc.M601337200 